\title{
Neoadjuvant Docetaxel, Cisplatin and Fluorouracil followed by Concurrent Cisplatin Intensity Modulated Radiation Therapy in Treatment of Locally Advanced Nasopharyngeal Carcinoma
}

\author{
E. Ibrahim ${ }^{1}$ and H. Zagloul ${ }^{2}$
}

\section{${ }^{1}$ MedicalOncology and ${ }^{2}$ Radiation Oncology Departments, King Fahad Specialist Hospital and Dammam, Saudi Arabia}

\begin{abstract}
Aim of the Study: To assess the tolerability, toxicity and efficacy of neoadjuvant Docetaxel, Cisplatin and Fluorouracil (TPF) followed by concurrent Cisplatin and radiotherapy (RT) in patients with locorgionally advanced nasopharyngeal carcinoma (NPC).

Patients and Methods: Twenty patients with locally advanced nasopharyngeal carcinoma who presented to the Oncology Centre at King Fahd Specialist Hospital, Dammam in Saudi Arabia received induction chemotherapy with 2 cycles of TPF chemotherapy: Docetaxel: $75 \mathrm{mg} / \mathrm{m}^{2}$, Cisplatin: $75 \mathrm{mg} / \mathrm{m}^{2}$ and continuous infusion of 5-Fluorouracil: $750 \mathrm{mg} / \mathrm{m}^{2}$ CI for 96 hours followed 3-4 weeks later by concurrent weekly Cisplatin (40 mg $/ \mathrm{m}^{2}$ ) and IMRT (Intensity Modulated Radio Therapy) (GTV:70 Gy over 35 fractions).

Results: Response to neoadjuvant chemotherapy was as follows: 6 patients $(30 \%)$ and 12 patients $(60 \%)$ achieved complete remission (CR) and partial remission (PR), respectively. One patient (5\%) had no response to induction chemotherapy and one patient (5\%) died post first cycle of chemotherapy. At last follow up, 17 patients $(85 \%)$ were in complete remission while 2 patients $(10 \%)$ had progressive disease. The most common acute toxicity of TPF was grade 3-4 neutropenia (40\%). The most common acute toxicity of Cisplatin plus RT was grade 3-4 mucositis (60\%). At last follow up (May 2012) seventeen patients (85\%) were alive and free of disease.

Conclusions: Neoadjuvant Docetaxel, Cisplatin and Fluorouracil (TPF) followed by concurrent Cisplatin and radiotherapy (RT) in patients with locorgionally advanced nasopharyngeal carcinoma (NPC) was feasible and resulted in complete remission rate of $85 \%$ with acceptable toxicity profile.
\end{abstract}

Key words: nasopharyngeal carcinoma, neoadjuvant chemotherapy, and radiotherapy.

Corresponding Author: Ehab Ibrahim E-mail: ehabhas@hotmail.com

\section{INTRODUCTION}

Radiotherapy is the primary treatment modality for all locally and regionally confined stages of nasopharyngeal carcinoma (NPC). Whereas the control of early-stage disease with radiotherapy is usually successful, the response of locally and regionally advanced NPC to radiation is poor because of local relapse and distant metastases ${ }^{1-4}$.

Diagnosis is made by biopsy and workup which includes careful examination, documentation of the size and location of the neck nodes; evaluation of cranial nerve function and hearing; skull films (especially skull base views), evaluating neural foramina; complete computed tomographic (CT) scan or magnetic resonance imaging (MRI) with views delineating the upper and lower extent of the lesion; chest $\mathrm{x}$-ray; hemogram; and chemistry panel ${ }^{5-7}$.

Major prognostic factors adversely influencing outcome of treatment include large size of the tumor, higher $\mathrm{T}$ stage, and the presence of involved neck nodes ${ }^{8}$.

The American Joint Committee on Cancer (AJCC) has designated staging by TNM classification to define nasopharyngeal cancer ${ }^{9}$.

Incorporation of chemotherapy with standard RT has improved the therapeutic outcome of patients with locoregionally advanced NPC. This is supported by a meta-analysis of six randomized trials suggesting that when compared with RT alone, the addition of chemotherapy in any sequence increases disease-free survival by $35 \%$ at 2 to 4 years and overall survival by $20 \%$ at 3 to 4 years ${ }^{10}$. A key question remains regarding the optimal sequencing of chemotherapy and RT. Many published randomized trials of concurrent chemoradiotherapy have demonstrated a progressionfree (PFS) and/or overall survival (OS) benefit over RT 
alone ${ }^{11-13}$. In this regard, neoadjuvant chemotherapy may have better tolerability, and moreover impressive tumor response rates have been reported in phase II studies ${ }^{14}$.

\section{MATERIAL AND METHODS}

Twenty patients with locally advanced nasopharyngeal carcinoma (stage III-IVB) with no distant metastasis who presented to the Oncology Centre at King Fahd Specialist Hospital, Dammam in Saudi Arabia were enrolled in this study during the period between July 2007 and April 2012. All patients received two cycles of TPF induction chemotherapy: Docetaxel: $75 \mathrm{mg} / \mathrm{m}^{2}$, Cisplatin: $75 \mathrm{mg} / \mathrm{m}^{2}$ and continuous infusion of 5-Fluorouracil: $750 \mathrm{mg} / \mathrm{m}^{2} \mathrm{CI}$ over 96 hours followed 3-4 weeks later by concurrent weekly Cisplatin (40 mg $/ \mathrm{m}^{2}$ ) and IMRT (GTV:70 Gy over 35 fractions). All patients had a non-metastatic disease as proved by doing chest and abdominal CT scan, bone scan and PET/CT scan. In this study, tumor staging was based on the American Joint Committee on Cancer (AJCC) criteria for NPC 9 .

\section{Patient Monitoring and Follow up:}

Before treatment, patients underwent a complete history and physical examination, including evaluation of performance status, assessment for the presence of concurrent co-morbid conditions with estimation of body weight, height, vital signs and measurement of palpable or visual lesions. Laboratory studies included a complete blood count (CBC) with white blood cell differential count, biochemistry profile, kidney function test, liver function tests, hepatitis markers, random blood sugar and Epstein-Barr virus (EBV) DNA assay. Radiological examinations, including CT head and neck, chest, abdomen and pelvis, were required before starting treatment, at the end of induction chemotherapy, post concurrent chemoradiation and then periodically. Isotopic bone scan was not done routinely. PET/CT was done at presentation and post induction chemotherapy. Patients should have dental clearance before initiation of radiation therapy.

During therapy, performance status, weight, vital signs and full blood counts were obtained before each chemotherapy cycle. In addition a complete medical history, including assessment of subjective and objective toxicity according to the WHO Common Toxicity Criteria (CTC) was obtained before the start of each new cycle s-16. $^{\text {15 }}$.

The primary end point of this study was response rate (RR). The treatment response was categorized according to Response Evaluation Criteria in Solid Tumors (RECIST) ${ }^{17}$ The secondary end point was to assess the toxicity and tolerability of chemotherapy.

\section{Chemotherapy Protocol:}

Neoadjuvant chemotherapy consisted of two cycles of TPF regimen: Docetaxel $\left(75 \mathrm{mg} / \mathrm{m}^{2}\right.$ administered intravenously [IV] over 1 hour), Cisplatin $(75 \mathrm{mg} /$ $\mathrm{m}^{2}$ administered intravenously [IV] over 1 hour) and continuous infusion of 5-Fluorouracil $\left(750 \mathrm{mg} / \mathrm{m}^{2}\right.$ CI over 96 hours) given on days 1 and 21. Docetaxel infusion was preceded by IV Dexamethasone 20 $\mathrm{mg}$, Chlorpheniramine $50 \mathrm{mg}$, Ranitidine $50 \mathrm{mg}$ and Granisetron $1 \mathrm{mg}$. During RT, cisplatin at a dose of $40 \mathrm{mg} / \mathrm{m}^{2}$ IV infusion was administered weekly for 7 weeks, given at approximately 60 minutes before receiving $\mathrm{RT}$.

\section{Radiation Therapy Technique:}

Intensity Modulated Radiation Therapy (IMRT) using Simultaneous Integrated Boost (SIB) was used in treating patients, three planning target volumes (PTVs) were created: PTV 70 Gy to the primary and involved nodes, PTV 60 Gy to rest of nasopharynx, the oropharynx, posterior two thirds of the anterior maxillary sinuses and to non involved upper neck nodes and finally PTV 54 Gy to non involved lower neck nodes.

\section{RESULTS}

From July 2007 to April 2012, 20 patients referred to Oncology Centre at King Fahd Specialist Hospital, Dammam in Saudi Arabia were enrolled in this study. Patients and tumor characteristics are shown in Table 1. The mean age was 42 years with a range of 17-64 years and SD 13.2. Fourteen patients (70\%) were males, while 6 patients $(30 \%)$ were females. Performance status of ECOG (Eastern Cooperative Oncology Group) (0-1) and (2) was present in 14 patients $(70 \%)$ and 6 patients $(30 \%)$, respectively. Five patients $(25 \%)$ in the study presented with tumor stage T2, 3 patients (15\%) presented with T3 stage and 12 patients $(60 \%)$ with T4 disease. One patient $(5 \%)$ had no lymph node metastasis at presentation, 1 patient $(5 \%)$ with N1 disease, 9 patients (45\%) with N2 disease and 9 patients $(45 \%)$ with N3 disease. The main symptom at presentation was neck swelling which was present in 14 patients $(70 \%)$. Other symptoms were headache, ear symptoms and epistaxis.

Overall, toxicity related to neoadjuvant chemotherapy with Docetaxel, Cisplatin and Fluorouracil was manageable. Induction chemotherapy was generally well tolerated. Nineteen patients completed the planned treatment, and were assessable for response and toxicity. Three patients (15\%) had a dose delay of $\geq 7$ days, because of delayed neutrophil recovery. Grade 3-4 neutropenia occurred in eight patients $(40 \%)$, while grade 3-4 thrombocytopenia 
without bleeding occurred in two patients (10\%). Three patients $(15 \%)$ developed uncomplicated, culture-negative grade 2 febrile neutropenia during induction chemotherapy. Grade 3-4 anorexia and nausea occurred in $60 \%$ of patients while, grade $3-4$ vomiting occurred in $15 \%$ of patients. Ninety percent of patients developed reversible grade 3 and 4 alopecia. Two patients (10\%) developed hypersensitivity reaction to Docetaxel during the first cycle which was mild and manageable. One patient $(5 \%)$ developed reversible renal impairment. Treatment-related death was encountered in one patient during induction chemotherapy.

Concurrent Cisplatin with radiation was tolerable and of accepted toxicity profile. The most commonly reported grade 3-4 toxicity were mucositis, dysphagia, skin reactions and weight loss which occurred in 60\%, $25 \%, 20 \%$ and $30 \%$, respectively, (Table 2 ).

The overall response rate to induction chemotherapy reached $90 \%$ with complete remission $(\mathrm{CR})$ and partial remission (PR) rates of $30 \%$ and $60 \%$, respectively. Six weeks post concurrent chemoradiation therapy, $85 \%$ and $10 \%$ of patients were in complete remission and partial remission, respectively, (Table 3 ).

Table 1: Patients and Characteristics.

\begin{tabular}{|c|c|c|}
\hline Characteristic & No & $\%$ \\
\hline \multirow{3}{*}{$\begin{array}{l}\text { Age, years } \\
\text { Median } \\
\text { Range }\end{array}$} & & \\
\hline & \multicolumn{2}{|c|}{42} \\
\hline & \multicolumn{2}{|c|}{$17-64$} \\
\hline Male: Female & $14: 6$ & $70: 30$ \\
\hline \multirow{3}{*}{$\begin{array}{l}\text { ECOG performance status } \\
\text { ECOG }(0-1) \\
\text { ECOG }(2)\end{array}$} & & \\
\hline & 14 & 70 \\
\hline & 6 & 30 \\
\hline \multirow{4}{*}{$\begin{array}{l}\text { T stage } \\
\text { T2 } \\
\text { T3 } \\
\text { T4 }\end{array}$} & 5 & 25 \\
\hline & & \\
\hline & 3 & 15 \\
\hline & 12 & 60 \\
\hline \multirow{4}{*}{$\begin{array}{l}\text { Nodal stage N } \\
\text { N0 } \\
\text { N1 } \\
\text { N2 } \\
\text { N3 }\end{array}$} & 1 & 5 \\
\hline & 1 & 5 \\
\hline & 9 & 45 \\
\hline & 9 & 45 \\
\hline
\end{tabular}

Table 2: Toxicity of Induction Chemotherapy.

\begin{tabular}{lcc}
\hline Type of Toxicty & No & \% \\
\hline Neutropenia & 8 & 40 \\
\hline Thrombocytopenia & 2 & 10 \\
\hline Febrile Neutropenia & 2 & 10 \\
\hline Anorexia \& Nausea & 12 & 60 \\
\hline Vomiting & 3 & 15 \\
\hline Alopecia & 18 & 90 \\
\hline Renal Impairment & 1 & 5 \\
\hline
\end{tabular}

Table 3: Treatment response.

\begin{tabular}{lcccc}
\hline $\begin{array}{l}\text { Mode of } \\
\text { Assessment }\end{array}$ & \multicolumn{2}{c}{$\begin{array}{c}\text { 6 Weeks Post } \\
\text { Induction } \\
\text { Chemotherapy }\end{array}$} & $\begin{array}{c}\text { 6 Weeks Post } \\
\text { Concurrent CRT }\end{array}$ \\
\cline { 2 - 5 } & No & $\%$ & No & \% \\
\hline CR & 6 & 30 & 17 & 85 \\
\hline PR & 12 & 60 & 2 & 10 \\
\hline
\end{tabular}

CR: Complete Remission, PR: Partial Remission.

\section{DISCUSSION}

Patients with locorgionally advanced NPC have traditionally been treated solely with conventional RT; however, many develop local and/or distant failures and the long term survival rates were not satisfactory. In an attempt to improve treatment outcomes, several groups have incorporated chemotherapy adjuvantly, neoadjuvantly or concurrently with RT in randomized controlled trials ${ }^{11-13,18-20,22,23}$ The results of a metaanalysis of 1528 patients from six randomized trials have shown that the addition of chemotherapy to radical RT for locorgionally advanced NPC increased both disease free/progression free and overall survival by between 20 and $40 \%$ at 2-4 years after treatment ${ }^{10}$.

The primary rationale for induction or adjuvant chemotherapy in nasopharyngeal cancer has been to decrease the risk of developing distant metastases. Promising 4-5 years OS rates exceeding 75\% have been reported in studies from Australia and North America using sequential neoadjuvant chemotherapy and chemoradiotherapy. Al-Amro et al. ${ }^{21}$ used two cycles of Cisplatin and Epirubicin followed by concomitant RT at $66 \mathrm{~Gy}$ in $2 \mathrm{~Gy}$ fractions and concurrent Cisplatin for three cycles $25 \mathrm{mg} / \mathrm{m}^{2}$ for 4 days on Days 42, 63, and 84. In another study, Hong et al. ${ }^{24}$ used three cycles of induction chemotherapy with Mitomycin, Epirubicin, Cisplatin, Fluorouracil, and Leucovorin (MEPFL) before RT. 
Three large trials of induction without concurrent chemotherapy have not demonstrated improved overall survival compared with radiation alone ${ }^{25-27}$ Chan et al. used induction chemotherapy of 2 cycles of Taxol (Paclitaxel) and Carboplatin followed by concurrent Cisplatin $\mathrm{RT}^{28}$. Another study used induction chemotherapy of 2 cycles of Docetaxel and Cisplatin followed by concurrent Cisplatin with radiotherapy ${ }^{29}$.

In our study induction chemotherapy was generally well tolerated and of acceptable toxicity profile. Ten percent of patients developed grade 3-4 anaemia. Wee et al. in 2003 reported grade 3-4 anaemia of 3\% in their study ${ }^{30}$. Grade 3-4 neutropenia occurred in $40 \%$ of patients. Our results are lower than what reported in 2001 by Hong et al. ${ }^{24}$ who reported an incidence of $59 \%$ of grade 3-4 neutropenia in his study. Al-Amro et al. ${ }^{21}$ and Chan et al. ${ }^{28}$ reported an incidence of grade 3-4 neutropenia of $31 \%$ and $36 \%$ respectively. This could be explained by the low myelotoxic potential of the regimen they used. Our results are much less than that reported by Chan et al. ${ }^{29}$ who reported an incidence of grade 3-4 neutropenia of $100 \%$ who used the same regimen of induction chemotherapy but without fluorouracil. One patient experienced grade 3-4 febrile neutropenia post first cycle of induction chemotherapy and died because of complications. Ninety percent of our patients developed grade 3-4 alopecia. Chan et al. ${ }^{28}$ did not report any grade 3-4 alopecia in his study.

Chemotherapy related toxicity during concurrent Cisplatin with radiation was seen in $60 \%$ of our patients who developed grade 3-4 mucositis within the radiation field which concurs with the results of Chan et al. ${ }^{28}$ and Lee et al. ${ }^{31}$ who reported an incidence of $55 \%$ and $62 \%$ of grade 3-4 mucositis, respectively. Al-Amro et $a .^{21}$ reported an incidence of grade 3-4 mucositis of $49 \%$.

The overall response rate (CR and PR) to neoadjuvant Taxotere, Cisplatin and Fluorouracil in the study was $90 \%$. Chan et al. ${ }^{28}$ reported an overall tumor response of $97 \%$. Chan et al. ${ }^{29}$ reported an overall response rate to neoadjuvant chemotherapy of $86 \%$. Hong et $a .^{24}$ reported complete remission rate of $45.5 \%$, while Paccagnella et al. $^{32}$ reported very low incidence of complete remission rate post induction chemotherapy which was $6.5 \%$ compared to $30 \%$ in our study.

In our study, 6 weeks post concurrent chemoradiation $85 \%$ of patients were in complete remission. Chan et al. ${ }^{28}$ reported complete remission rates of $100 \%$ in his study, while Paccagnella et al. ${ }^{32}$, Cho et al. $^{33}$ and Yamouni et al. ${ }^{34}$ reported complete remission rates of $50 \%$ and $48 \%$ and $76.8 \%$, respectively.
E. Ibrahim and H. Zagloul

The current study confirms the feasibility of combining neoadjuvant TPF (Taxotere- CisplatinFluorouracil) followed by concurrent chemoradiotherapy in patients with advanced NPC and resulted in a complete remission rate $(\mathrm{CR})$ in $85 \%$ of patients. Patient tolerability and compliance to neoadjuvant TPF chemotherapy was acceptable.

\section{CONCLUSION}

Neoadjuvant chemotherapy using Docetaxel, Cisplatin and Fluorouracil (TPF) followed by concurrent Cisplatin-RT used for patients with locally advanced nasopharyngeal cancer results in excellent tumor control, manageable toxicity profile and achieving complete remission rate of $85 \%$.

\section{REFERENCES}

1. Perez CA, Devineni VR, Marcial-Vega V, Marks JE, Simpson JR, Kucik N. Carcinoma of the nasopharynx: Factors affecting prognosis. Int.J.Radiat.Oncol.Biol.Phys. 1992;23(2):271-80.

2. Zhang EP, Lian PG, Cai KL, Chen YF, Cai MD, Zheng XF, et al. Radiation therapy of nasopharyngeal carcinoma: Prognostic factors based on a 10-year follow-up of 1302 patients. Int.J.Radiat.Oncol.Biol.Phys. 1989;16(2):301-5.

3. Qin DX, Hu YH, Yan JH, Xu GZ, Cai WM, Wu XL, et al. Analysis of 1379 patients with nasopharyngeal carcinoma treated by radiation. Cancer 1988;61(6):1117-24.

4. Lee AW, Poon YF, Foo W, Law SC, Cheung FK, Chan DK, et al. Retrospective analysis of 5037 patients with nasopharyngeal carcinoma treated during 19761985: Overall survival and patterns of failure. Int.J.Radiat. Oncol.Biol.Phys. 1992;23(2):261-70.

5. Mendenhall WM, Riggs CEJ, Cassisi NJ. Treatment of head and neck cancers. In: DeVita VT, Hellman S, Rosenberg SA, editors. Cancer: Principles and Practice of Oncology. Edition 7 Philadelphia: Lippincott Williams \& Wilkins; 2004. pp. 662-732.

6. Laramore GE. Radiation therapy of head and neck cancer. Berlin: Springer-verlag; 1989.

7. Cummings $\mathrm{CW}$, Fredrickson JM, Harker LA. Otolaryngology - head and neck surgery. Saint Louis: Mo: Mosby-Year Book, Inc; 1998.

8. Sanguineti G, Geara FB, Garden AS, Tucker SL, Ang KK, Morrison WH, et al. Carcinoma of the nasopharynx treated by radiotherapy alone: Determinants of local and regional control. Int.J.Radiat.Oncol.Biol.Phys. 1997;37(5):985-996.

9. Pharynx (including base of tongue, soft palate and uvula. American Joint Committee on Cancer:: AJCC Cancer Staging Manual. 6th ed. New York, NY: Springer; 2002. pp. 31-46.

10. Huncharek M, Kupelnick B. Combined chemoradiation versus radiation therapy alone in locally advanced nasopharyngeal carcinoma: Results of a meta-analysis of 1,528 patients from six randomized trials. Am.J.Clin.Oncol. 2002;25(3):219-23. 
11. Al-Sarraf M, LeBlanc M, Giri PG, Fu KK, Cooper J, Vuong $\mathrm{T}$, et al. Chemoradiotherapy versus radiotherapy in patients with advanced nasopharyngeal cancer: Phase III randomized Intergroup study 0099. J.Clin.Oncol. 1998;16(4):1310-7.

12. Chan AT, Teo PM, Ngan RK, Leung TW, Lau WH, Zee $\mathrm{B}$, et al. Concurrent chemotherapy-radiotherapy compared with radiotherapy alone in locoregionally advanced nasopharyngeal carcinoma: Progression-free survival analysis of a phase III randomized trial. J.Clin. Oncol. 2002;20(8):2038-44.

13. Lin JC, Jan JS, Hsu CY, Liang WM, Jiang RS, Wang WY. Phase III study of concurrent chemoradiotherapy versus radiotherapy alone for advanced nasopharyngeal carcinoma: Positive effect on overall and progression-free survival. J.Clin.Oncol. 2003;21(4):631-7.

14. Teo PM, Chan AT. Treatment strategy and clinical experience. Semin.Cancer Biol. 2002;12(6):497-504.

15. National Cancer Institute (NCI). Guidelines for reporting of adverse drug reactions. Bethesda, Md.: NCI, Division of Cancer Treatment, Cancer Therapy Evaluation Program; 1988.

16. WHO. WHO handbook for reporting results of cancer treatment. 1979. WHO, Geneva.

17. Therasse P, Arbuck SG, Eisenhauer EA, Wanders J, Kaplan RS, Rubinstein L, et al. New guidelines to evaluate the response to treatment in solid tumors. European Organization for Research and Treatment of Cancer, National Cancer Institute of the United States, National Cancer Institute of Canada. J.Natl.Cancer Inst. 2000;92(3):205-16.

18. Chua DT, Sham JS, Choy D, Kwong DL, Au GK, Kwong PW, et al. Patterns of failure after induction chemotherapy and radiotherapy for locoregionally advanced nasopharyngeal carcinoma: The Queen Mary Hospital experience. Int.J.Radiat.Oncol.Biol.Phys. 2001;49(5):1219-28.

19. Rossi A, Molinari R, Boracchi P, Del Vecchio M, Marubini E, Nava M, et al. Adjuvant chemotherapy with vincristine, cyclophosphamide, and doxorubicin after radiotherapy in local-regional nasopharyngeal cancer: Results of a 4-year multicenter randomized study. J.Clin. Oncol. 1988;6(9):1401-10.

20. Al-Sarraf M, Le Blanc M, Giri P. Superiority of five year survival with chemo-radiotherapy (CT-RT) vs radiotherapy in patients with locally advanced nosapharyngeal cancer (NPC). Intergroup (0099) (SWOG 8892, RTOG 8817, ECOG 2388 phase III study: Final report. Proceedings of the American Society of Clinical Oncology 2001;20: 227a.

21. Al-Amro A, Al-Rajhi N, Khafaga Y, Memon M, AlHebshi A, El-Enbabi A, et al. Neoadjuvant chemotherapy followed by concurrent chemo-radiation therapy in locally advanced nasopharyngeal carcinoma. Int.J.Radiat.Oncol. Biol.Phys. 2005;62(2):508-13.
22. Wolden SL, Zelefsky MJ, Kraus DH, Rosenzweig KE, Chong LM, Shaha AR, et al. Accelerated concomitant boost radiotherapy and chemotherapy for advanced nasopharyngeal carcinoma. J.Clin.Oncol. 2001;19(4):1105-10.

23. Cheng SH, Yen KL, Jian JJ, Tsai SY, Chu NM, Leu SY, et al. Examining prognostic factors and patterns of failure in nasopharyngeal carcinoma following concomitant radiotherapy and chemotherapy: impact on future clinical trials. Int.J.Radiat.Oncol.Biol.Phys. 2001;50(3):717-26.

24. Hong RL, Ting LL, Ko JY, Hsu MM, Sheen TS, Lou PJ, et al. Induction chemotherapy with mitomycin, epirubicin, cisplatin, fluorouracil, and leucovorin followed by radiotherapy in the treatment of locoregionally advanced nasopharyngeal carcinoma. J.Clin.Oncol. 2001;19(23):4305-13.

25. Webb A, Cunningham D, Scarffe JH, Harper P, Norman A, Joffe JK, et al. Randomized trial comparing epirubicin, cisplatin and fluorouracil versus fluorouracil, doxorubicin and methotrexate in advanced esophagogastric cancer. J.Clin.Oncol. 1997;15(1):261-7.

26. Cvitkovic E, Eschwege F, Rahal M, Dosen M,Z., Krajina Z, Armand JP. Preliminary results of a randomized trial comparing neoadjuvant chemotherapy (cisplatin, epirubicin, bleomycin) plus radiotherapy vs. Radiotherapy alone in stage IV $(\leq \mathrm{N} 2, \mathrm{M} 0)$ undifferentiated nasopharyngeal carcinoma: A positive effect on progression-free survival. Int.J.Radiat.Oncol.Biol.Phys. 1996;35(3):463-9.

27. Chan AT, Teo PM, Ngan RK. A phase III randomized trial comparing concurrent chemotherapy-radiotherapy with radiotherapy alone in locoregionally advanced nasopharyngeal carcinoma. Proc.Am.Soc.Clin.Oncol. 1988;19:415a (abstr 1637).

28. Chan AT, Ma BB, Lo YM, Leung SF, Kwan WH, Hui EP, et al. Phase II study of neoadjuvant carboplatin and paclitaxel followed by radiotherapy and concurrent cisplatin in patients with locoregionally advanced nasopharyngeal carcinoma: Therapeutic monitoring with plasma Epstein-Barr virus DNA. J.Clin.Oncol. 2004;22(15):3053-60.

29. Chan AT, Hui EP, Ma B, Leung SF, King A, Mo F, et al. A randomized phase II study of concurrent cisplatin-radiotherapy (RT) with or without neoadjuvant chemotherapy using docetaxel and cisplatin in advanced nasopharyngeal carcinoma (NPC). J.Clin.Oncol.(Meeting Abstracts) 2005;23(16S):Abstract.no. 5544.

30. Wee J, Tan EH, Tai BC, Wong HB, Leong SS, Tan T, et al. Randomized trial of radiotherapy versus concurrent chemoradiotherapy followed by adjuvant chemotherapy in patients with American Joint Committee on Cancer/ International Union against cancer stage III and IV nasopharyngeal cancer of the endemic variety. J.Clin. Oncol. 2005;23(27):6730-8. 
31. Lee AW, Lau WH, Tung SY, Chua DT, Chappell R, $\mathrm{Xu} \mathrm{L}$, et al. Preliminary results of a randomized study on therapeutic gain by concurrent chemotherapy for regionally-advanced nasopharyngeal carcinoma: NPC9901 Trial by the Hong Kong Nasopharyngeal Cancer Study Group. J.Clin.Oncol. 2005;23(28):6966-75.

32. Paccagnella A, Buffoli A, Koussis H, Gava A, Franceschi T, Gardani G, et al. Concomitant chemoradiotherapy (CT/RT) vs neoadjuvant chemotherapy with docetaxel/cispaltin/5fluorouracil (TPF) followed by CT/RT in locally advanced head and neck cancer. Final results of a phase II randomized study. J.Clin.Oncol.(Meeting Abstracts) 2008;26(15S):6000.
33. Cho S, Bae W, Hwang J, Shim H, Lee J, Lim S, et al. Phase II study of docetaxel, cisplatin and 5-FU induction chemotherapy followed by concurrent chemoradiotherapy for advanced nasopharyngeal cancer. J. Clin. Oncol. (Meeting Abstracts) 2008;26(15S):17010

34. Yamouni M, Beldjilali Y, Benhadji KA, Khellafi H, Betkaoui F, Kaid Y, et al. A phase II trial of induction chemotherapy with cisplatin, docetaxel, and capecitabine followed by concurrent cisplatin-radiotherapy in advanced nasopharyngeal carcinoma. J.Clin.Oncol.(Meeting Abstracts) 2011;29 Suppl:(abstr 5543). 